East African Medical Journal Vol. 80 No. 1. January 2003

PNEUMOCYTIS CARINII PNEUMONIA IN HIV/AIDS PATIENTS AT AN URBAN DISTRICT HOSPITAL IN KENYA

J.M. Chakaya, MBChB, MMed(Nbi) DThM (London), Chest Physician/Principal Research Officer, CRDR, KEMRI, P.O. Box 47855, Nairobi, Kenya, C. Bii, Msc, Research officer, CMR, KEMRI, P.O. Box 19464, Nairobi, Kenya, L. Ng'ang'a, PhD, Principal Research Officer, CRDR, KEMRI, P.O. Box 47855, Kenya, E. Amukoye, MBChB, MMed (Nbi), Senior Research Officer, CRDR, KEMRI, P.O. Box 47855, Nairobi, Kenya, T. Ouko, HND, Laboratory Technologist, CMR, KEMRI, P.O. Box 19464, Nairobi, L. Muita, HND, Laboratory Technologist, CRDR, KEMRI, P.O. Box 47855, Nairobi, Kenya, S. Gathua, MBChB, MMed, DTCD(Cardiff), Chest Physician, Menelik Medical Centre, P.O. Box 55164, Nairobi, Kenya, J. Gitau, MBChB, MMed, Radiologist, CCR, KEMRI, P.O. Box 20778, Nairobi, I. Odongo, MBChB, MMed, Chest Physician, Mbagathi District Hospital, P.O. Box 20725, Nairobi, Kenya, J.M. Kabanga, Dip Lab Tech, Laboratory Technologist, CCR, KEMRI, P.O. Box 20778, Nairobi, Kenya, K. Nagai, MD, PhD, Paediatrician, School of Medicine, Sapporo Medical University, S1, W16, Chuo-ku, Sapporo, Hokkaido 060-8543, Japan, S. Suzumura, MD, PhD, Teine Royal Hospital, Sapporo Japan, Y. Sugiura, MD, PhD, Bureau of International Medical Centre of Japan, 1-21-1 Toyama, Shinjuku-ku, Tokyo, 162-8655, Japan

Request for reprints to: Dr. J.M. Chakaya, Centre for Respiratory Diseases Research, Kenya Medical Research Institute, P.O. Box 47855, Nairobi, Kenya

\title{
PNEUMOCYTIS CARINII PNEUMONIA IN HIV/AIDS PATIENTS AT AN URBAN DISTRICT HOSPITAL IN KENYA
}

\author{
J.M. CHAKAYA, C. BII, L. NG’ANG’A, E. AMUKOYE, T. OUKO, L. MUITA, S. GATHUA,
} J. GITAU, I. ODONGO, J.M. KABANGA, K. NAGAI, S. SUZUMURA and Y. SUGIURA

\begin{abstract}
Background: Pneumocytis carinii pneumonia has generally been regarded to be an uncommon opportunistic infection in HIV infected individuals in sub-Saharan Africa. The reason for this has not been clear but postulates included a lack of suitable pathogenic types in the African environment, diagnostic difficulties and the more commonly held belief that African HIV infected individuals were dying early from common non-opportunistic pathogens before severe degrees of immunosuppression occured. Recently a trend has emerged at the Mbagathi district hospital whereby an increasing number of HIV infected patients are empirically treated for Pneumocytis carinii pneumonia (PCP) based on clinical and radiological features.
\end{abstract}

Objective: To determine the prevalence of PCP and clinical outcomes of HIV infected patients presenting at the Mbagathi District Hospital, Nairobi with the presumptive diagnosis of PCP.

Setting: Mbagathi District Hospital, a 169-bed public hospital in Nairobi, Kenya.

Methods: Patients presenting with a sub-acute onset of cough and dyspnoea were eligible for the study if they were found to have bilateral pulmonary shadows and had negative sputum smears for AFBS. Consenting patients who had no contraindication to fiberoptic bronchoscopy had a clinical evaluation which was followed with a fiberoptic bronchoscopy procedure where bronchoalveolar lavage fluid (BALF) was obtained. BALF was examined for cysts of $P$. carinii using toluidine blue stain and immunofluorescent antibody test (IFAT). BALF was also processed for fungi, bacteria and mycobacteria using routine procedures. Standard treatment with high dose cotrimoxazole was offered to all patients who were then followed up until discharge from hospital or death whichever came first.

Results: Between June 1999 and August 2000 a total of 63 patients were referred for bronchoscopy. Of these four declined to undergo the fiberoptic bronchoscopy procedure, four died before the procedure could be done, one was judged too sick to undergo the procedure and three had been on cotrimoxazole for longer than five days. Thus 51 patients underwent bronchoscopy. Pneumocytis carinii stain was positive in 19 (37.2\%) while death occured in $16(31.4 \%)$ of the 51 patients. There were more deaths in those without PCP but this difference was not statistically significant (odds ratio 0.68 (95\% CI 0.35-1.32; $P=0.2$.

Conclusion: PCP was found to be common in HIV infected patients presenting with clinical and radiological features of the disease. The mortality rate for patients with a presumptive diagnosis of $\mathrm{PCP}$ is high. This study suggests that cotrimoxazole preventive therapy may be a useful intervention in symptomatic HIV infected patients in Kenya for the prevention of PCP and may avert deaths from this disease.

\section{INTRODUCTION}

Pneumocytis carinii pneumonia (PCP) has generally been considered rare in HIV infected adults in sub-Saharan Africa including $\operatorname{Kenya(1-6).~This~}$ situation has been the complete opposite of what prevailed in North America and Europe as the Acquired Immunodeficiency Syndrome (AIDS) epidemic 
unfolded. AIDS was actually recognised about two decades ago following the appearance of this previously rare form of pneumonia in homosexual men in the United States of America(7,8). As the AIDS epidemic progressed PCP became the most prominent opportunistic infection and was observed in about $80 \%$ of HIV/AIDS patients resident in the United States of America $(9,10)$. This observation led to the widespread adoption of preventive therapy against PCP with guidelines established on when to institute this therapy(11). PCP was however not completely unknown in Africa. The disease had been described, in Congolese infants(12) and Ugandan children with severe malnutrition(13) in the pre-HIV era. The postulates for the rarity of this disease in adult HIV infected patients in Africa ranged from absence of the pathogen(14), diagnostic difficulties to the more widely held belief that African AIDS patients were dying early from common and more virulent pathogens before severe degrees immunossuppression developed(3). Recent observations from Zimbabwe indicate that the prevalence of PCP may be increasing $(15,16)$. In Kenya there has been very limited data on the prevalence of PCP in HIV/AIDS patients. In the only published study from Kenya, Rana et al. found two cases of PCP out of nine patients in a post mortem series(17). In both patients PCP was found to be present together with another infection, the most common of these being cytomegalovirus and tuberculosis.

Despite the described rarity of PCP in African HIV/AIDS patients, clinicians have increasingly been treating patients empirically for this disease based on compatible clinical and/or radiological features. Whether this practise was justified, considering the toxicity of the treatment and the need for long-term secondary chemoprophylaxis implied by choosing to treat was unclear. This study was therefore conducted to a) determine the prevalence of PCP in hospitalised patients with clinical and radiological features compatible with the disease and in whom PCP treatment was being considered or had just commenced, and b) Describe clinical outcomes in patients treated for PCP at the Mbagathi District Hospital, Nairobi.

\section{MATERIALS AND METHODS}

Study site: The Mbagathi district hospital is a small, 169-bed public hospital in Nairobi established in 1956 and which initially served as a specialized infectious disease hospital with an emphasis on tuberculosis until 1995 when it became a general medical and paediatric hospital. Despite the change in official status the hospital has continued to serve as an infectious diseases hospital with a high proportion of adult patients having HIV related illnesses including TB (Chakaya, personal observation).

Study population: Known HIV infected patients were selected for PCP evaluation if they had a cough with shortness of breath and bilateral shadows on the chest X-ray in the absence of positive sputum smears for Acid Fast Bacilli (AFB). All patients admitted at the Mbagathi district hospital with features of HIV disease or a severe infection are to date routinely offered diagnostic HIV counselling and testing. The HIV tests are done using rapid diagnostic tests kits supplied by the National STD and AIDS Control programme (NASCOP). Various such kits, including capillus and immunocomb, were in use during the period of the study. Patients are reported as positive when the results of two different rapid tests are positive. Tests for degree of immunosuppression (CD4/CD8) and viral load are not available in this hospital and the study was not able to carry them out for cost considerations. An informed written consent was obtained before the patients underwent fiberoptic bronchoscopy. The study was granted scientific and ethical approval by the Kenya Medical Research Institute.

Fiberoptic Bronchoscopy: Selected patients were assessed for suitability for fiberoptic bronchoscopy. Those who did not consent or were considered too sick to undergo the procedure were excluded. Patients were instructed to fast for at least four hours before the procedure. Pre-medication consisted of $0.6 \mathrm{mg}$ atropine given intravenously half an hour before the procedure and 2.5 to $5 \mathrm{mg}$ of intravenous midazolam (Dormicum, Astra Zeneca) at the time of the procedure. The anaesthetic procedure involved spraying the nose and oropharynx with $10 \%$ lidocaine (Xylocaine, Astra Zeneca) and the vocal cords, trachea and the main bronchi with $2 \mathrm{ml}$ aliquots of $2 \%$ lignocaine to a maximum of $20 \mathrm{mg}$. The fiberoptic bronchoscope (Olympus BF type IT 30 or Olympus BF type P30, Olympus, Japan) was introduced usually via the nasal route or sometimes the mouth. After a thorough inspection of the airways the scope was lodged into the segment judged to have the densest shadows on the chest $\mathrm{X}$-ray or the right middle lobe or lingular segment of the left upper lobe in those with diffuse radiographic shadows. Twenty $\mathrm{ml}$ aliquots of warm sterile saline, to a total of $60 \mathrm{ml}$ were instilled and gently sucked back. The obtained bronchoalveolar lavage fluid (BALF) was immediately sent to the laboratory for microbiological analysis. Transbronchial biopsies were not done because it was felt doing them would be too dangerous in these very sick patients especially in the absence of adequate resuscitation facilities and facilities for chest tube placement and drainage.

\section{Microbiological Processing of BALF}

Stain for Pneumocytis carinii: Pneumocytis carinii was detected in BALF using Toluidine Blue Stain and immunofluorescent staining.

Modified Toluidine Blue O Staining Procedure: Toluidine blue O procedure(18) was used for the detection of cysts of P. carinii in BALF. In brief BALF was placed in a centrifuge tube, and centrifuged at $200 \mathrm{x}$ g for 15 minutes. Then the supernatant less $5 \mathrm{ml}$ was removed and the sediment plus the remaining, supernatant was transferred to a clean centrifuge and gently mixed with a Pasteur pipette. Slides were made from this material stained and examined using $20 \mathrm{X}$ or $40 \mathrm{X}$ objectives. A known positive control slide was included for each patient's specimen.

Immunofluorescent stain: Detection of P. carinii from BALF was done using an immunofluorescent test kit (Shield 
Diagnostics Ltd, Dundee, U.K). The procedure was done according to the manufacturer's instructions and the specimen was considered positive when there were five or more oocysts seen.

Other Microbiological Investigations: Bronchoalveolar lavage fluid also underwent routine culture for bacteria and fungi. Briefly primary isolation was done on sheep blood agar (SBA), chocolate-agar (CHO), Bromothymol blue (BTB) agar and sabouraud dextrose agar (SDA). Quantitative culture was employed in SBA and $\mathrm{CHO}$. SBA and $\mathrm{CHO}$ were incubated at $35^{\circ} \mathrm{C}$ under $5 \%$ carbon dioxide for 18 to 24 hours. BTB was incubated in air at $35^{\circ} \mathrm{C}$ for $18-24$ hours while SDA was incubated for up to four weeks. Identification of suspected bacterial and fungal pathogens was done using analytical profile index (API strips biomerieux) in addition to slide culture for the identification of the moulds. Bacterial drug susceptibility testing was performed as per National Committee for Clinical Laboratory Standards (NCCLS 1997). The presence of a bacterial agent at $10^{4}$ colony forming units (cfu) per $\mathrm{ml}$ of BALF was considered aetiologically relevant. Often multiple therapies were used on these patients and therefore it was difficult to use response to treatment as indicator of the relevance of any bacterial organism isolated.

Statistical Analysis: Mean differences of clinical symptoms between those with and those without PCP were compared using student t-test (unpaired), while differences in proportions were parametrically compared using normal distribution. The $95 \%$ confidence interval of odds ratio about patients prognosis between those with and without PCP was parametrically calculated using normal distribution. A p-value of $5 \%$ was defined as the significant level.

\section{RESULTS}

Between June 1999 and August 2000 a total of 63 patients were referred for bronchospy. Of these four declined to undergo the fiberoptic bronchoscopy procedure, four died before the procedure could be done, one was judged to be too sick to undergo the procedure and three had been on cotrimoxazole for longer than five days. Thus a total of 12 patients were excluded from the study. The results presented therefore are from the 51 patients who underwent the bronchoscopy procedure. Of the 51 patients studied $19(38 \%)$ were male and $31(62 \%)$ female. The median age was 30.0 years with a range of 20.0 to 56.0 years. PCP was found in $14(27.4 \%)$ and $19(37.2 \%)$ of the 51 patients studied using toluidine blue $\mathrm{O}$ and IF staining respectively. Cough and dyspnoea were the most common symptoms. Cough was present in 18 $(94.7 \%)$ of 19 patients with PCP and in $26(92.9 \%)$ of 28 patients without PCP. Similarly dyspnoea was present in $18(94.7 \%)$ of 19 patients with PCP and in $26(92.9 \%)$ of 28 patients without PCP. There were no statistically significant differences in the frequency of symptoms between those with and those without PCP(Table 1).
Table 1

Clinical symptoms and signs in patients with presumed $P C P$

\begin{tabular}{lccc}
\hline Symptom & $\begin{array}{c}\text { PCP+ (n=19) } \\
\text { No.(\%) }\end{array}$ & $\begin{array}{c}\text { PCP-(n=32) } \\
\text { No.(\%) }\end{array}$ & p-value \\
\hline Dyspnoea & $18(94.7)$ & $26(81.3)$ & 0.176 \\
Fever & $15(78.9)$ & $21(65.6)$ & 0.312 \\
Wheeze & $10(52.6)$ & $11(34.3)$ & 0.200 \\
Cough & $18(94.7)$ & $26(81.3)$ & 0.326 \\
Wasting & $13(68.4)$ & $20(62.5)$ & 0.669 \\
Respiratory rate & $51.7(18.2)$ & $50.4(16.5)$ & 0.800 \\
Mean(SD) & & & \\
Temperature & $36.8(0.71)$ & $37.0(0.82)$ & 0.847 \\
Mean (SD) & & & \\
Systolic BP & $102(8.3)$ & $106.0(11.8)$ & 0.208 \\
Mean (SD) & & & \\
Diastolic BP & $63.2(4.5)$ & $64.0(7.4)$ & 0.648 \\
Mean (SD) & & & \\
Bronchial sounds & $3(15.8)$ & $8(25.0)$ & 0.349 \\
Crackles & $11(57.9)$ & $15(46.9)$ & 0.447 \\
Cyanosis & $3(15.8)$ & $4(12.5)$ & 0.741 \\
Oral thrush & $7(36.8)$ & $15(46.9)$ & 0.484 \\
Skin rash & $5(26.3)$ & $5(15.6)$ & 0.352 \\
\hline
\end{tabular}

Table 2

Other pathogens isolated from patients with presumed $P C P$

\begin{tabular}{lcc}
\hline Pathogen & $\begin{array}{r}\text { PCP negative } \\
(\mathrm{n}=32)\end{array}$ & $\begin{array}{c}\text { PCP positive } \\
(\mathrm{n}=19)\end{array}$ \\
\hline Klebsiella pneumoniae & 4 & 2 \\
Pseudomonas aeruginosa & 1 & 0 \\
Staphylococcus aureus & 4 & 2 \\
Haemophilus influenzae & 0 & 1 \\
Norcardia spp & 1 & 0 \\
Corynebacterium spp & 0 & 1 \\
Escherichia coli & 1 & 0 \\
Cryptococcus neoformans & 1 & 0 \\
Fuserium spp & 0 & 1 \\
S. aureus plus K. pneumoniae & 1 & 0 \\
S. aureus plus Enterobacter & 1 & 0 \\
\hline
\end{tabular}

Only a small range of these other pathogens were isolated from BALF probably because these patients had all received or were receiving one or more antibiotics prior to the bronchoscopy procedure. The organisms isolated included; Corynebacterium spp, Escherichia coli, Haemophilus influenzae, Norcadia spp, Pseudomonas aeruginosa, Cryptococcus neoformans, Fuserium spp in one patient each, Staphylococcus aureus in three patients and Klebsiella Pneumoniae in six patients (Table2).

The clinical outcome of the 51 patients studied is shown in Table 3. Of the 19 patients with proven PCP, five $(26.3 \%)$ died versus $11(34.4 \%)$ of 32 patients without PCP. 
Table 3

Clinical outcomes of patients with presumed PCP

\begin{tabular}{|c|c|c|c|}
\hline Condition & $\begin{array}{l}\text { Death } \\
\text { No. }(\%)\end{array}$ & $\begin{array}{l}\text { Discharge } \\
\text { No. }(\%)\end{array}$ & $\begin{array}{l}\text { Odds ratio } \\
\text { for death }\end{array}$ \\
\hline $\mathrm{PCP}(\mathrm{n}=11)$ & $3(27.2)$ & 8 (72.7) & 1(Reference) \\
\hline $\begin{array}{l}\text { PCP plus other } \\
\text { pathogens } \\
(\mathrm{n}=8)\end{array}$ & $2(25.0)$ & $6(75.0)$ & $0.9(\mathrm{CI}: 0.1-7.1)$ \\
\hline $\begin{array}{l}\text { Other pathogens } \\
\quad(\mathrm{n}=13)\end{array}$ & $6(46.1)$ & 7 (54.9) & 2.3(CI:0.4-12.7) \\
\hline $\begin{array}{r}\text { No microbiological } \\
\text { diagnosis }(n=18)\end{array}$ & $5(27.8)$ & $13(72.2)$ & $1.0(0.2-5.5)$ \\
\hline Total $($ No. $=51)$ & $16(31.4)$ & 35 (68.6) & \\
\hline
\end{tabular}

This difference was not statistically significant (odds ratio $0.68,95 \%$ CI 0.35-1.32; $\mathrm{P}=0.2$ ). All patients referred for bronchoscopy were started on high dose cotrimoxazole either within five days prior or immediately after the bronchoscopy procedure.

\section{DISCUSSION}

This study demonstrates that PCP is a common disease in HIV/AIDS patients admitted to this small public hospital with clinical and radiological features compatible with the disease. As in the rest of subSaharan Africa PCP has been considered an uncommon or non-existent opportunistic infection in HIV/AIDS patients in Kenya(1-6). There has been very scanty data to indicate that this situation is changing. Two studies from Zimbabwe done a few years apart suggested that the prevalence of PCP in HIV infected patients with pneumonia unresponsive to penicillin had increased from $22 \%$ in the late eighties to $33 \%$ in $1992(15,19)$. In Kenya, the general clinical impression was that PCP was rare in HIV infected individuals and this impression is supported by unpublished clinical observations. For example PCP was found in only two of the 100 patients who underwent fiberoptic bronchoscopy at the Mbagathi district hospital between 1988 and 1990 (Gathua, personal communication). Thus the current study suggests that the prevalence of PCP at the Mbagathi District Hospital has increased significantly in the last ten or so years. Our study had however major limitations and therefore should not be misinterpreted as being proof of an increase in the prevalence of PCP in HIV/ AIDS patients at this hospital. The major limitation of the study was a selection bias. Not all patients with pulmonary disease of unknown aetiology were included in the study. The study restricted itself to only those patients where a presumptive diagnosis of PCP had been made from clinical and radiological criteria by the clinicians directly involved in the clinical care of these patients. These criteria were not standardized and were not based on previous studies where sensitivity or specificity of the criteria had been determined. Thus it is possible that many patients with PCP were left out because the clinicians did not think of the disease or the presentation was atypical. However the study was a pragmatic approach to assess what had been observed as an increasing trend in this hospital to treat patients with cough and shortness of breath for PCP if they had bilateral pulmonary shadows and negative sputum smears. The study may have influenced clinicians to make the diagnosis of PCP more often so that their patients with problematic lung pathology could get the bronchoscopy procedure done. Thus our results can only be applicable to the group of patients with cough and shortness of breath who have bilateral pulmonary shadows and negative sputum smears and may not reflect the true prevalence of the disease in HIV infected patients with lung disease. The other limitation of our study is the small sample size. However despite these limitations our study has documented for the first time in Kenya that PCP is an important and common opportunistic infection in HIV infected persons. A prospective study to obtain incidence data and the associated factors for PCP is certainly warranted. If the incidence of PCP is actually increasing the reason for this development, just like the earlier observation of the rarity of PCP in Africa is unclear. Diagnostic difficulties cannot quite explain the previous observation of the rarity of PCP. The post mortem studies carried out in Uganda(2) and Cot D' Ivoire(6) for example were as intensive a search for the disease as there can be, yet no PCP was found. Thus the absence of the disease in the early years of the HIV epidemic in Africa was probably real. It is possible that African HIV/AIDS patients were actually not living long enough to develop opportunistic infections like PCP which has been observed to occur in those with severe degrees of immunosuppression. There are however no studies that have demonstrated that African HIV/AIDS patients were dying when their immune status is relatively well preserved. Improved survival however remains the most plausible explanation for the increased commonness of PCP that this study reveals. There has been almost two decades of experience in the clinical care of HIV infected patients in Africa which may have resulted in better survival of HIV infected patients. This hypothesis however may be impossible to prove.

Our study indicates that PCP is a serious illness that carries significant mortality. We were not able to perform CD4 T-lymphocyte counts and therefore we cannot state the degree of immunosupression in our patients. However most of our patients had clinical features compatible with severe immune dysfunction. Future studies should attempt to correlate the occurrence of this disease with degree of immunosuppression as measured by CD4 T-lymphocyte counts. In the industrialized world, PCP is a disease that is largely restricted to those with CD4 T-lymphocyte counts 
below 200/ul and therefore primary prophylaxis for PCP is recommended for those with this level of CD4 cell count(11). There is no data to indicate that the same level of CD4 count is applicable in African HIV/ AIDS patients. Since most patients with HIV infection in Kenya do not get to have their CD4-T cell count assessed, other criteria will have to be used for the primary prevention of the disease. These criterias are likely to be confined to clinical parameters. Certainly the disease seems to be common and severe enough for the adoption of widespread preventive therapy to be recommended. Cotrimoxazole is the drug of choice for prevention and treatment of $\mathrm{PCP}(11)$. There are several studies from Africa that indicate that cotrimoxazole preventive therapy (CPT) is efficacious in protecting HIV infected patients from severe HIV related morbidity and mortality(19-21). The favoured dose is $960 \mathrm{mg}$ given daily and it seems that the patients who benefit most are those with severe degrees of immunosuppression. Most protection form CPT is speculated to accrue from the prevention of bacterial infections including pneumococcal disease and disease by non-typhi Salmonella. This was one reason why CPT was initially received with some scepticism in parts of Africa, Kenya included, where most isolates of these organisms are resistant to cotrimoxazole. The results of our study would support the adoption of CPT in Kenya may be more for the prevention of diseases like PCP and toxoplasmosis than for the prevention of common bacterial infections.

Fiberoptic bronchoscopy is generally unavailable in Kenya except in Nairobi, the capital city. Similarly the less sensitive but still useful procedure of sputum induction using inhalation of hypertonic saline is also not available. The examination of spontaneously expectorated sputum has such low sensitivity that it is not recommended for the routine diagnosis of $\mathrm{PCP}(22)$. Thus diagnostic difficulties will continue to hamper the optimal management of this potentially treatable condition in Kenya. There is need therefore to develop simple clinical and radiological diagnostic criteria that may be used by clinicians to diagnose this disease even in the absence of bronchoscopy. At the moment the best recommendation that can be made is that PCP treatment should be considered in those HIV infected patients developing cough and shortness of breath especially in the presence of diffuse bilateral pulmonary shadows except when an alternative diagnosis has been made and the patient seems to be responding well to specific treatment.

The slightly improved prognosis of patients with PCP as compared with those without PCP found in this study may be explained by the effect of specific therapy against PCP offered by high dose cotrimoxazole.

We conclude that PCP is a common disease in HIV infected patients presenting with cough, shortness of breath and bilateral pulmonary shadows at the Mbagathi District Hospital. The case fatality of patients with presumed PCP is high but specific therapy against PCP using high dose cotrimoxazole may offer some protection against death. The rest of our study support the adoption of PCP preventive therapy with cotrimoxazole in symptomatic HIV infected patients especially in the presence of clinical evidence of severe immunosuppression.

\section{ACKNOWLEDGEMENTS}

This study was supported by Japan International Cooperation Agency (JICA) through the Research and Control of Infectious and Parasitic Diseases Project, a technical cooperation project by the Government of Japan to the Government of the Republic of Kenya. This study could not have been done without the support of health care staff at the adult medical ward of the Mbagathi District Hospital. Ms. Judy RaugaGichu is acknowledged for her data entry work. The paper is published with the permission of the Director, KEMRI.

\section{REFERENCES}

1. Ellvin, K.M., Lumbwe, C.M., Luo, N.P., Bjorkman, A., Kallenius, G. and Linder, E. Pneumocytis carinii is not a major cause of pneumonia in HIV infected patients in Lusaka Zambia.Trans. Roy. Soc. Trop. Med. \& Hyg. 1989; 83:553-555.

2. Lucas, S.B., Goodgame, R., Koejan, G. and Serwadda, D. Absence of pneumocytosis in Ugandan AIDS patients. AIDS. 1989; 3:47-48.

3. Lucas, S.B. Missing Infections in AIDS. Trans. Roy. Soc. Trop. Med. \& Hyg. 1990; 84(Supp/1):34-38.

4. Lucas, S.B., Odida, M. and Wabinga, H. The pathology of severe morbidity and mortality caused by HIV infection in Africa. AIDS. 1991; 5(Suppl 1):S143-S148.

5. Carme, J., Mboussa, J.M., Andzin, M., Mbouni, P., Mpele, P. and Datry, A. Pneumocytis carinii is rare in AIDS in Central Africa. Trans. Roy. Trop. Med \& Hyg. 1991; 85:80.

6. Abouya, Y., Beaumel, A., Lucas, S., et al. Pneumocystis carinii pneumonia. An uncommon cause of death in African patients with acquired immunodeficiency syndrome. Ame. Rev. Respir. Dis. 1992; 145:617-620.

7. Masur, R., Micheles, M.A., Greene, J. et al. An outbreak of community acquired Pneumocytis carinii Pneumonia: Initial manifestation of acquired cellular immune dysfunction. 1981; 305:1431-4383.

8. Gottlieb, M.S., Schroff, R., Shanker, H.M. Pneumocytis carinii pneumonia and mucosal candidiasis in previously healthy homosexual men: evidence of a new acquired cellular immunodeficiency. New Eng. J. Med. 1981; 305: 1425-1431.

9. Update: acquired immunodeficiency syndrome-United States. MMWR Morb. Mortal Wkly. Rep. 1986; 35:757-766.

10. Update: acquired immunodeficiency syndrome-United States. MMWR Morb Mortal Wkly Rep 1986; 35:17-21.

11. 1997 USPHS/IDSA guidelines for the prevention of opportunistic infection in persons infected with human immunodeficiency virus: disease specific recommendations. Clin. Infect. Dis. 1997; 25(suppl)3:S313-S335.

12. Thijis, A. and Janssens, P. Pneumocytosis in Congolese infants. Trop. Geogr. Med. 1963; 15:158-172. 
13. Bwibo, N. and Owor, R. Pneumocystis carinii pneumonia in Ugandan African children. W. Afr. Med. J. 1970; 19: 184-185.

14. Lesley G. and Lucas, S.B. Does Pneumocytis carinii occur in Kenya. Trans. Roy. Soc. Trop. Med \& Hyg. 1982; 76:198-199.

15. Adam, S.M., Lovemore, K.Z.G., Susanne, K. et al. Pneumocytis carinii pneumonia in Zimbabwe. Lancet 1995; 346:1258-1261.

16. David, A. R. and Joseph, A.K. Pneumocytis carini in Africa an emerging pathogen? Commentary. Lancet. 1995; 346:1242-1243.

17. Rana, H., M.P., Meme, H.K., Chakaya, J.M. et al. Autopsy findings in HIV-1 infected adults in Kenya (Letter). J. Acq. Imm. Defi. Syndro. Human Retrov. 1997; 14: 83-85.

18. Gosey, L.L., Howard, R.M., Whebsky, F.G. et al. Advantages of modified toluidine blue $\mathrm{O}$ stain and bronchoalveolar lavage for the diagnosis of pneumocytis carinii pneumonia. J. Clin. Microbiol. 1985; 22:303-807.
19. Madeod, D.T., Neil, P., Robertson, U.J. et al. Pulmonary diseases in patients infected with the human immunodeficiency virus in Zimbabwe, Central Africa. Trans. Roy. Soc. Trop. Med \& Hyg. 1989; 83:94-697.

20. Xavier, A., Genevieve, C., Alain, A. et al. Early chemoprophylaxis with trimethoprim sulphamethoxazole for HIV 1 infected adults in Abdijan, Cote d'Ivoire: a randomised trial. Lancet. 1999; 353:1463-1168.

21. Stefan, Z.W., Madeleine, S.M., Allison, D. et al. Efficacy of trimethoprim sulphamethoxazole prophylaxis to decrease morbidity and mortality in HIV 1 infected patients with tuberculosis in Abdijan, Cote d'Ivoire: a randomised controlled trial. Lancet. 1999; 353:1469-1475.

22. Badri, M., Ehrlich, R., Wood, R. and Maartens, G. Initiating co-trimoxazole prophylaxis in HIV infected patients in Africa; an evaluation of the provisional WHO/UNAIDS recommendations. AIDS. 2001;15:1143-1148.

23. Robert F. M. and David, M. Pneumocytis carinii pneumonia. Thorax. 1995; 50:191-200.

\section{ANNOUNCEMENT}

\section{KENYA MEDICAL ASSOCIATION 31ST ANNUAL SCIENTIFIC CONFERENCE AND ANNUAL GENERAL MEETING}

Hosts: Kenya Medical Association - Mombasa Division

Venue: Travellers Beach Hotel, Mombasa

Date: $\quad 23 r d-27$ th April, 2003

Theme: Current Trends in Treatment and Prevention of HIV/AIDS and other Diseases in sub-Saharan Africa

For further details and registration contact:

Dr. Gordon Peter T.K. Yossa

Scientific Conference Secretary

Office: KMA Office, Pandya Memorial Hospital

Nyerere Avenue

P.O. Box 83178, Mombasa

E-mail: kmamsa@ikenya.com

Fax: (011) 316482 (through Pandya Hospital)

Tel: (011) 230979 or $314140 / 1,229252$ ext. 148

N.B: Send abstracts to the Conference Secretariat 